\title{
Puncture-Proof Picture Archiving and Communication System
}

\author{
Charles E. Willis, Charles W. McCluggage, Michael R. Orand, and Bruce R. Parker
}

As we become increasingly dependent on our picture archiving and communications system (PACS) for the clinical practice of medicine, the demand for improved reliability becomes urgent. Borrowing principles from the discipline of Reliability Engineering, we have identified components of our system that constitute single points of failure and have endeavored to eliminate these through redundant components and manual work-around procedures. To assess the adequacy of our preparations, we have identified a set of plausible events that could interfere with the function of one or more of our PACS components. These events could be as simple as the loss of the network connection to a single component or as broad as the loss of our central data center. We have identified the need to continue to operate during adverse conditions, as well as the requirement to recover rapidly from major disruptions in service. This assessment led us to modify the physical locations of central PACS components within our physical plant. We are also taking advantage of actual disruptive events coincident with a major expansion of our facility to test our recovery procedures. Based on our recognition of the vital nature of our electronic images for patient care, we are now recording electronic images in two copies on disparate media. The image database is critical to both continued operations and recovery. Restoration of the database from periodic tape backups with a 24-hour cycle time may not support our clinical scenario: acquisition modalities have a limited local storage capacity, some of which will not contain the daily workload. Restoration of the database from the archived media is an exceedingly slow process, that will likely not meet our requirement to restore clinical operations without significant delay. Our PACS vendor is working on concurrent image databases that would be capable of nearly immediate switchover and recovery.

Copyright $\odot 2001$ by W.B. Saunders Company

$\mathbf{P}$

HYSICIANS IN electronic imaging hospitals depend on their picture archiving and communication system (PACS) in much the same way that a Grand Prix driver depends on his tires during

From the Department of Radiology; Baylor College of Med. icine, Houston; and Edward B. Singleton Diagnostic Imaging Senices, Texas Children's Hospital, Houston, TX.

Address reprint requests to Charles $E$. Willis, $P h D, E d w a r d$ L. Singleton Diagnostic Imaging Services, Texas Children's Hospital, 6621 Fannin MC 2-2521, Houston, TX 77030-2399. E-mail: cewillis@texaschildrenshospital.org.

Copyright 02001 by IV.B. Saunders Company

0897.1889/01/1402-1017\$35.00/0

doi:10.1053/jdim.2001.2382I a race. Like the crew chief at LeMans, our goal is to provide, within reason, a safe environment for an inherently unsafe activity: Only recently have reports begun to appear in the literature concerning measures to enhance the reliability of PACS. ${ }^{1}$ Original thinking on uptime requirements for PACS was borrowed largely from experience with acquisition modalities, rather than from reliability analysis of a complete, large-scale electronic imaging system. The early history of PACS is dominated by efforts just to make the systems work, rather than to make them work 24 hours a day, 7 days a week, 365 days a year. It appears that initial thinking on reliability needs to be revised based on actual experience in the field, where continuity of clinical care must be maintained.

\section{SINGLE POINTS OF FAILURE}

A key to improving reliability of a system is to identify and eliminate any component that constitutes a single point of failure. Beyond the simple consequence of a local loss of service, failure of one of these components would have global impact on the system. Identifying single points of failure and assessing their impact requires an understanding of the architecture of the system. Early thinking in PACS design acknowledged "critical components" that would require higher reliability and better service response than "noncritical components." Critical components may be considered a subset of the collection of single points of failure. For example, the image database is usually considered a critical PACS component. Utilities, such as electrical power, are not even considered as PACS components; however, their loss can cripple the PACS system just as effectively as the loss of a critical component. Table 1 lists single points of failure for our PACS.

\section{Redundancy}

Redundancy is a strategy for eliminating single points of failure. The overall failure rate of a system of components functioning in parallel is the product of the individual failure rates. A system composed of two components in parallel that each fails $1 \%$ of the time, will only fail $0.01 \%$ of the time. A system composed of the same two components in series will fail $2 \%$ of the time, because 
Table 1. Single Points of Failure

1. Image database (OS)

2. Radiology Information System (RIS)

3. RIS interface

4. Hospital Information System (HIS)

5. Web Server

6. Network Interface Adapter (NIA)

7. Network Gateway (NG)

8. Archive Server (AS)

9. Magneto Optical Disk (MOD) media

10. Main Data Center

11. Hospital Broadband Network

the system failure rate is the sum of the failure rates of individual components connected in series.

It is interesting to note that one device which brought PACS out of the speculative environment and into the real world was the redundant array of inexpensive disks (RAID), which itself improves on the reliability of a single disk. Recently, redundant PACS archive server technology was also demonstrated.

Emergency power, or generator power, is an example of redundancy that is built into hospitals to avoid the consequences of a loss of utility electrical power. Emergency power is usually available only for a limited number of devices, so it is important to consider how many PACS components need to operate during a general power outage. Uninterruptible power supplies (UPS) are a standard feature included in data centers, but is often an afterthought for PACS clients outside of the core components. These are often necessary as power conditioners during the brief transition from utility to generator power, or to allow an orderly shutdown that preserves the disk file structure of client.

A large-scale PACS that includes multiple similar acquisition modalities and display stations affords some inherent "distributed redundancy." Operators might have to travel to a different floor of the hospital to process images, or travel to another care unit to view images, which is inconvenient and undesired, but allows clinical operations to continue uninterrupted.

\section{Asynchronous Operations}

Even during an outage of a critical component, some parts of the PACS can continue to operate for some period. For example, if an interruption in service prevents archiving of images, acquisition may still continue until the limited local storage capacity for that modality is exceeded.

\section{Manual Workaround}

A manual workaround is an alternative strategy for avoiding the consequences of a single point failure of an automated system. Workarounds, or "downtime procedures," are intended to allow continued clinical operations for a limited time until automated service is restored. ${ }^{2}$ Acquisition using conventional film screen and interpretation from hard-copy images are examples of workarounds of last resort.

\section{Recovery}

After service is restored and nominal operations can resume, it is critical to bring the automated system up-to-date on processes that occurred during the outage. This recovery process may include transmission of images that were acquired during the outage.

\section{FAILURE MODES AND EFFECTS ANALYSIS}

To determine where resources should be directed to improve reliability, it is important to identify a set of plausible disruptive events that could interfere with one of more PACS components. These events could be as localized as the loss of a network connection to a single PACS client, or as broad as the loss of our entire PACS data center. An analysis of the effects of these events coupled with an assessment of the need to continue to operate during such adverse conditions and the time allowed to recover after the event dictates reasonable limits on contingency provisions. For example, it is unnecessary to plan for a disaster that would permanently destroy the physical plant of the hospital, because we would have no further requirement to conduct imaging operations. On the other hand, a regional natural disaster such as a hurricane that might cause temporary damage to the physical plant, flooding, and long power outages, must be considered by a hospital in our region. We considered both natural and manmade disasters. In assessing provisions for continuity of operations, it is more important to focus on the affected PACS component rather than on the external event that caused the loss. Flooding caused by either a rainstorm or a utility failure could have equivalent effects on PACS compo- 
Table 2. Disaster Scenarios

1. Loss of a single image or exam

2. Loss of a single piece of media (MOD or tape)

3. Loss of a single core component (NG, AS, OS, RIS interface, or web server)

4. Loss of a single display component (review station or laser camera)

5. Loss of a single NIA

6. Loss of a single QA Workstation (PS, or VIPS)

7. Loss of a single acquisition device (CR, DF, MR, CT, US, FD)

8. Loss of power to the datacenter

9. Loss of network connectivity to the datacenter

10. Loss of HVAC to the datacenter

11. Loss of physical access to the datacenter

12. Loss of all components in the datacenter

13. Relocation of the datacenter

14. Interruption of service during major upgrade of software requiring database migration

15. Loss of RIS connection

nents. Table 2 provides a list of scenarios that interfere with PACS operations.

\section{RELIABILTY IMPROVEMENT PLAN}

Based on the results of our failure modes and effects analysis, we identified a need to continue to operate under adverse conditions and to recover rapidly from any major disruption in service. This assessment led us to modify the quantity, location, and configuration of many PACS components within our healthcare enterprise.

\section{Computed Radiography Clusters}

In both our main $\mathrm{x}$-ray department and our busy outpatient imaging center, we have two computed radiography (CR) scanners side-by-side. While one unit is out of service, the other carries the full workload. We also have duplicate quality-control (QC) workstations and multiple identification (ID) stations at each location. Each of these departments has a networked laser camera, in the event that hard-copy output is needed.

Individual CR scanners are deployed in our inpatient imaging areas, but each has its own QC workstation, ID station, and dry-process laser camera. All of the clients in a cluster are connected to the same network switch, so that in the event of a loss of connectivity to the central PACS components, the CR cluster can continue clinical operations.

\section{Secondary Data Center}

We have now established a secondary data center that will contain all components necessary to operate the PACS independently of the primary data center at reduced performance, that is, an archive server, network gateway, additional image database, radiology information system (RIS) interface, and Web Server. All images are now recorded in duplicate on disparate media (MOD and helical tape) in two different locations. ${ }^{3} \mathrm{Al}-$ though our secondary data center is located on another floor of our hospital in an unused computed tomorgraphy (CT) computer room, there is no reason that it could not be located offsite, given a high-speed network connection. ${ }^{4}$ When the secondary center is out of operation, all images are available in the primary center via shelf management of MOD media.

\section{Independent Outlying Health Centers}

Each of our two health centers that acquire CR examinations has its own scanner, QC workstation, and review station. The review station is configured with its own gateway process and includes an image database that periodically updates the central image database via a dedicated $\mathrm{Tl}$ connection. In this way, each health center can function asynchronously from the main hospital.

\section{Niche PACS in Ultrasound and Nuclear Medicine}

In our Ultrasound Department, we went from direct Digital Imaging and Communications in Medicine (DICOM) connections with our PACS archive to a stand-alone ultrasound niche-PACS connected to our main PACS archive via a DICOM gateway. Although the change in topology was dictated by a requirement to enable dedicated workstations for ultrasound viewing, it affords the Ultrasound service the ability to continue to function during service interruptions in the main PACS.

Nuclear Medicine has always operated asynchronously from the main PACS with a connection though a DICOM gateway. Recently, the Nuclear Medicine niche-PACS has been updated by the substitution of the DICOM gateway and the addition of a third camera and two dedicated workstations. 


\section{Multiple Gateway's}

Our PACS contains two other types of gateways. Medical gateways act as laser camera servers and as network interface adapters for our oldest $\mathrm{CR}$ scanners. Although the medical gateways can handle multiple inputs and outputs, they are configured so that loss of a single unit does not stop all production from a given modality. Medical gateways had another important function as analog-todigital converters, before we converted all our modalities to digital interfaces. Input to a nonfunctional medical gateway can also be re-routed to another. Re-routing is also necessary during laser camera maintenance.

Network gateways are the "traffic cops" of the PACS. They receive images from acquisition modalities, verify RIS information, and route the images to workstations and archive stations. The network gateway function, previously running only on the archive servers, was relocated to four individual central processing units (CPU). Each acquisition modality has a primary network gateway destination and an altemate, for use in the event of problems with its primary.

\section{Capability to Produce and Interpret Hard-Copy Images}

We have deliberately maintained the capability to produce and review hard-copy images. This means maintaining some conventional cassettes, lightboxes and alternators, darkrooms, automatic processors, and film stocks. The film stocks have to be rotated periodically and the processors are activated only when needed. Film digitizers are needed to introduce a representative electronic image into the PACS image database upon recovery. This decision had little real cost during the transition from a film-based department to an electronic department, but has significant impact in planning new and expanded facilities. This cost must be weighed against the risk of not being able to conduct imaging operations.

\section{BAPTISM UNDER FIRE}

The continuous process of upgrading and expanding our PACS to accommodate filmless operations has presented a rich environment for challenging the reliability of our system. The current major expansion of our main hospital and replacement of our outpatient treatment center has af- forded us numerous opportunities to test our ability to continue imaging operations during disruptive events. A few of our battle scars are described below.

\section{Hospital-Wide Power Outages}

The first lesson we learned from repeated outages in electrical power was that we really did not know what devices were connected to emergency power. Next we learned that we had not defined the procedure and sequence for deliberately powering down PACS clients. Third, we learned that we had not provided a UPS for every device that required one, and that the UPS for a review station was not capable of powering both monitors and the CPU for any length of time. We also learned that electrical power without heating ventilation, and air conditioning (HVAC) is inadequate for computers and operators alike. We learned that devices outside our control, such as switches and routers in telecommunications closets, might also be affected by power interruptions, so we learned to be prepared for concurrent network outages. A final lesson was that some devices, notably the medical gateways, that appeared to withstand power and network interruptions, actually needed to be restarted in order to restore their normal function.

\section{Hospital Network Infrastructure Reconfiguration}

As our PACS has been growing, the hospital broadband network has been evolving. Coincident with the hospital expansion, the network infrastructure is changing to accommodate dynamic host configuration protocol (DHCP). All PACS clients will continue to have fixed internet protocol (IP) addresses, but will reside on a different subnet. Moving each of these clients causes an interruption in services, and requires modification of the host table on all clients. Once complete, our PACS clients should operate in greater isolation from extraneous network traffic. We are also upgrading all our central PACS components from Fast Ethernet to Gigabit Ethernet. This involves disconnection from the old service, installation of a new network interface card, and reconnection to the new service.

\section{Relocation of the Imaging Department}

In the expansion process, our main imaging department will be split between two floors of the 
hospital. This involves disconnecting and reconnecting many PACS clients.

\section{Relocation of Primary Data Center}

Our primary data center will move to occupy space vacated during the expansion. During the displacement, PACS services will be provided by the secondary data center.

\section{RIS Periodic Maintenance}

During RIS outages, demographic information cannot be verified by PACS. Examinations acquired during these periods can be manually annotated, but are automatically sequestered until RIS-verified. During long outages, RIS verification can be disabled, but this involves risk of corrupting the image database with inaccurate demographics. Exams scheduled and performed during the RIS downtime will not generate events that trigger important PACS functions such as the retrieval of relevant prior studies. When the RIS is restored, examinations are manually scheduled after the fact to reconcile RIS accounting.

\section{Software Upgrades}

Software upgrades should be considered planned service interruptions of unknown duration. Upgrades entail significant downtime, especially if they involve database migration. The anticipated result of each upgrade should be studied in advance. The upgrade should be performed when least inconvenient in terms of clinical operations. Proper function should be verified after the upgrade is complete, before the technical staff departs the hospital premises.

\section{Special Notes on the Image Database}

It is difficult to overstate the importance of the image database: it is absolutely critical to continued operations and recovery. The present method of preserving the database is to write a tape backup of the database every day. This tape is stored in a fireproof container outside the datacenter. There are seven individual tapes, one for each day of the week. In theory, the database can thus be restored to the point it was at most 24 hours in the past, or up to 48 hours in the past in the case of a media failure. In practice, the examinations that are the most critical are the most recent: this method does not preserve the most important entries. The entire database can be reconstructed from records on the physical media; however, this method is extremely time consuming and does not address our need for rapid restoration of services. At present we have two parallel image databases operating at two remote health centers that periodically reconcile with the main image database. These "rear-realtime" databases are not fully concurrent: there is some latency in their data and certain examination status information is not accurately received from the RIS interface.

A unique feature of our PACS cache management presents a significant problem for maintaining continuity of clinical operations in the face of service interruptions. Our database regards a disconnected client as the "least busy." Requests for examinations having images residing on the disconnected client go exclusively to that client, even when copies are available elsewhere on functioning clients. This process continues until manually interrupted with the removal of the disconnected client from the host table. Imagine the disruption of services when someone trips over the network cable to the workstation for case conferences. None of these interesting active cases can be viewed for patient care anywhere in the hospital until the workstation is deleted from the host table, or the cable is plugged back into the network. This is the major reason that we cannot use our clinical review stations in a portable mode, to support multiple conference rooms or to move between operating rooms.

\section{CONCLUSIONS}

PACS downtime can only be tolerated by providing altemative methods for acquiring, interpreting, and reporting diagnostic imaging examinations. Implementing workarounds involves fault detection, fault isolation, initiation of work-around, fault correction, and recovery, and notification. The effects of PACS downtime can be minimized by redundancy in design, well-defined workaround procedures, and rapid recovery. Improvements are needed in automatic detection and reporting of off-nominal operations; automatic rerouting of image traffic; transparent, synchronized image databases; and automatic recovery processes. 


\section{REFERENCES}

1. McBiles M, Chacko AK: Coping with PACS downtime in digital radiology. J Digit Imaging 13:136-142, 2000

2. Ramthun S, James EM, Hangiandreou N, et al: Electronic imaging system implementation at Mayo Clinic Rochester: Downtime procedures and communication plans. J Digit Imaging 13:206-207, 2000 (suppl 1)

3. Behlen FM, Sayre RE, Weldy JB, et al: "Permanent records": Experience with data migration in radiology information system and picture archiving and communication system replacement. J Digit Imaging 13:171-174, 2000 (suppl 1)

4. Avrin DE, Andriole KP, Yin L, et al: Simulation of disaster recovery of a picture archiving and communications system using off-site hierarchal storage management. J Digit Imaging 13:168-170, 2000 (suppl 1) 